\title{
Impact of previous diagnosis of gestational diabetes on lifestyle habits in the next pregnancy
}

\begin{abstract}
Gestational diabetes (GDM) is a serious condition predisposing both the mother and child to health complications. Key means for treatment are lifestyle related, primarily adherence to a healthy diet and increase in physical activity. The aim of the study was to evaluate dietary quality and physical activity in early pregnancy of women reporting history of GDM compared to healthy women participating in a population-based study. Pregnant women were enrolled to the study by announcements in social media. The interested women $(\mathrm{n}=1034)$ filled in an electronic questionnaire on their background data, validated Index of Diet Quality (IDQ) and index of leisure-time physical activity (MET-index) in early pregnancy. The protocol was approved by the Ethics Committee of the University of Turku, Finland. The study population characteristics were representative of the Finnish pregnant women as compared with values reported in national perinatal statistics, except for overrepresentation of primiparas (54\% compared to $41 \%)$ and underrepresentation of smokers during pregnancy $(2.2 \%$ compared to $13 \%)$. Of the multiparas, $18.3 \%$ reported having been diagnosed with GDM in a previous pregnancy, which is in accordance to that in the general population (19\%). Having a history of GDM was not reflected in the dietary quality in the present pregnancy. The IDQ score of the women with history of GDM (adj. mean 9.5) did not differ from those with no history (adj. mean 9.3, NS). When evaluating the categorized values, $45.8 \%$ of the women with the history of GDM and $45.4 \%$ of those without had a good dietary quality (IDQ score 10 or above). Similarly, the MET-index of the women with history of GDM (4.8 hrs/wk) did not differ from that of the women with no history (4.8 hrs/wk). Also, the categorized MET-index did not differ between the groups. The physical activity level of the women with history of GDM was light in the majority $(52 \%)$ of the women, moderate in $41 \%$ and vigorous in $7 \%$. Despite the known risks that GDM induces to health of the pregnant women and their babies, the dietary quality and physical activity of the women with a history of GDM did not differ from that of the women who were not previously affected. The results indicate that new means are needed alongside with the traditional counselling practices to motivate healthy lifestyle changes in pregnant women, particularly those at risk for recurrent GDM.
\end{abstract}

\section{Conflict of Interest}

The authors declare no conflict of interest. Support was received from Business Finland (\#3486/31/2015) to the University of Turku. The funding source had no role in the design, execution, analyses, interpretation of the data, or decision to submit results. 